$\operatorname{CONF}-970469--16$

\title{
A Review of a Method for Dynamic Load Distribution, Dynamical Modeling, and Explicit Internal Force Control When Two Manipulators Mutually Lift and Transport a Rigid Body Object
}

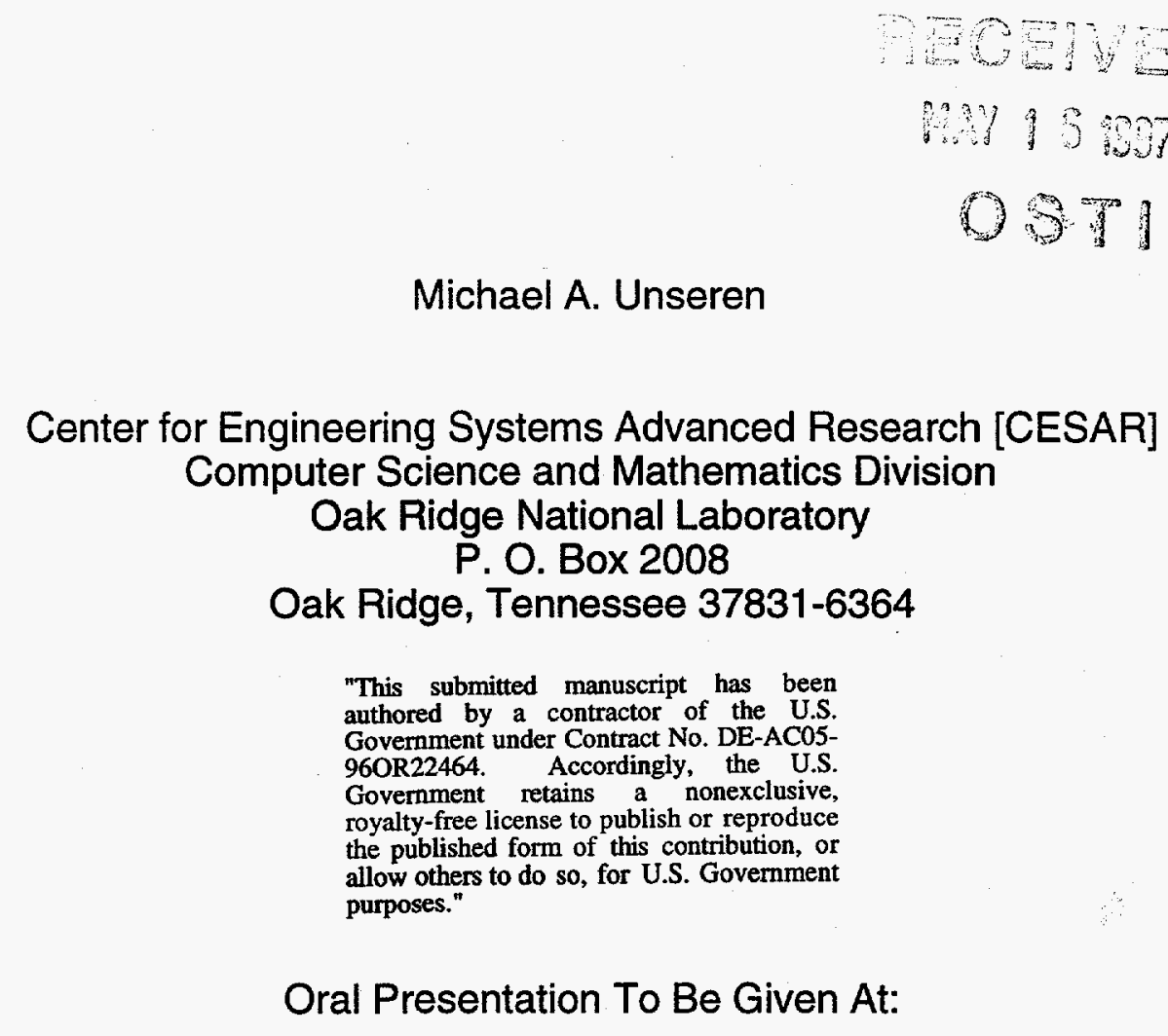

1997 IEEE International Conference on Robotics \& Automation Tutorial T2 - Modeling \& Control of Multi-Arm Robot Systems

Albuquerque, New Mexico

April 20, 1997

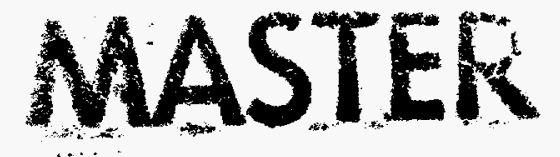

${ }^{*}$ Research sponsored by the Office of Engineering Research Program, Basic Energy Sciences, U.S. Department of Energy, under Contract No. DE-AC05-960R22464 with Lockheed Martin Energy Research Corp. 


\section{DISCLAIMER}

This report was prepared as an account of work sponsored by an agency of the United States Government. Neither the United States Government nor any agency thereof, nor any of their employees, make any warranty, express or implied, or assumes any legal liability or responsibility for the accuracy, completeness, or usefulness of any information, apparatus, product, or process disclosed, or represents that its use would not infringe privately owned rights. Reference herein to any specific commercial product, process, or service by trade name, trademark, manufacturer, or otherwise does not necessarily constitute or imply its endorsement, recommendation, or favoring by the United States Government or any agency thereof. The views and opinions of authors expressed herein do not necessarily state or reflect those of the United States Government or any agency thereof. 


\section{DISCLAMMER}

Portions of this document may be illegible in electronic image products. Images are produced from the best available original document. 


\title{
A Review of a Method for Dynamic Load Dis- tribution, Dynamical Modeling, and Explicit Internal Force Control When Two Manipula- tors Mutually Lift and Transport a Rigid Body Object $^{\dagger}$
}

\author{
Michael A. Unseren \\ Center for Engineering Systems Advanced Research \\ Computational Intelligence Group \\ Intelligent Systems Section \\ Computer Science and Mathematics Division \\ Oak Ridge National Laboratory \\ P.O. Box 2008 \\ Oak Ridge, TN 37831-6364
}

\begin{abstract}
The paper reviews a method for modeling and controlling two serial link manipulators which mutually lift and transport a rigid body object in a three dimensional workspace [29, $30,31,33]$. A new vector variable is introduced which parameterizes the internal contact force controlled degrees of freedom. A technique for dynamically distributing the payload between the manipulators is suggested which yields a family of solutions for the contact forces and torques the manipulators impart to the object. A set of rigid body kinematic constraints which restrict the values of the joint velocities of both manipulators is derived. A rigid body dynamical model for the closed chain system is first developed in the joint space. The model is obtained by generalizing our previous methods for deriving the model. The joint velocity and acceleration variables in the model are expressed in terms of independent pseudovariables. The pseudospace model is transformed to obtain reduced order equations of motion and a separate set of equations governing the internal components of the contact forces and torques. A theoretic control architecture is suggested which explicitly decouples the two sets of equations comprising the model. The controller enables the designer to de-
\end{abstract}

\footnotetext{
of Research Sponsored by the Office of Engineering Research Program, Basic Energy Sciences, U.S. Department of Energy, under Contract No. DE-AC05-960R22464 with Lockheed Martin Energy Research Corp.
} 
velop independent, non-interacting control laws for the position control and internal force control of the system.

\section{INTRODUCTION}

The problem of modeling and controlling two fixed base, serial link robotic manipulators to mutually lift and transport an object has been a subject of intensive study and research these past ten years. This interest has been motivated by the potential benefits of employing automatic and programmable two handed cooperative manipulation in diverse areas such as material handling and assembly. In the former application, two manipulators can cooperatively lift and transport large or voluminous objects that would be difficult or awkward for a single manipulator to move. Further, two cooperating manipulators can transport objects whose mass is beyond the lifting capacity of just one. Two cooperating manipulators can reduce the need for fixturing in many assembly applications, and may ultimately lead to fixtureless assembly in the air.

There have been numerous approaches proposed for modeling the interactions between the object and each manipulator and for controlling the forces and torques at the points of contact. In [1], models were developed which allow the contacts between the manipulators and object to be accidentally (e.g., due to slippage) or deliberately broken or the nature of the constraints changed due to wanted or unwanted disturbances. The analysis focused on a pair of two link planar revolute manipulators maintaining sliding point contacts with an object. The object was stabilized using a spring-dashpot combination.

In [2], it was proposed that a pair of six degree of freedom (DOF) manipulators maintain rolling point contacts with a rigid object. In the approach, three virtual revolute joints were added at the location of each effector. The kinematics of the rolling grasps was modeled.

The application of impedance control has resulted in successful implementations of two manipulators transporting an object $[3,4,5]$. These approaches enforce a controlled impedance of the manipulator endpoints or of the manipulated object itself.

This paper, however, focuses on the case of two serial link manipulators mutually lifting and transporting objects that are rigid and jointless in a three dimensional workspace under the assumption of there being no relative motion between the end effectors and the object. That is to say, it is assumed that each manipulator securely holds the object without any slippage. The manipulators and object form a single closed chain mechanism, and there exists a large body of literature on modeling and controlling the manipulators in this configuration [6-31]. It should be mentioned that there has been some results reported for the case of two manipulators holding objects consisting of two rigid bodies connected by passive rotary or spherical joints $[33,34]$, where the assumption of no relative motion between each end effector and the rigid body it holds still applied.

There are two challenging problems when modeling and controlling a dual manipulator closed chain system. First, the problem of dynamically distributing the load induced by the object between the manipulators is underspecified. Indeed, assuming that the object is rigid and jointless, its dynamical equations, i.e., Newton's and Euler's equations, are linear functions of the twelve components of contact force and torque the manipulators impart to it. Therefore, assuming that a reference trajectory for the center of mass of the object has been specified, there are infinitely many solutions for the contact forces and torques based on the object's dynamical equations. Each contact force ${ }^{\S}$ solution contains a component that causes the object to move along the reference trajectory and a component that induces internal stress and torsion in the object but does not contribute to its motion. Various approaches for distributing the load have been proposed $[7,13,17,18,19,20,26,27,28,29]$.

The second problem is how to control the motion of the closed chain system and the contact forces. It has been shown that a set of six rigid body kinematic constraints are imposed on the values of the joint variables of both manipulators in this configuration [31]. Each constraint causes a loss of one position controlled DOF. This complicates the motion

$0 \S$ Contact force implies both contact force and contact torque hereinafter, unless otherwise specified. 
control problem because the number of actuated joints exceeds the number of positional DOF in the closed chain. If each manipulator is kinematically nonredundant, then the motion control objective is object trajectory tracking. If at least one of the manipulators is redundant, then there are additional positional DOF available to satisfy other objectives [35].

Another part of the control problem involves controlling or influencing the values of the internal component of the contact forces. Left unregulated, the internal forces could assume large values that result in the manipulators pulling against each other and would require large actuation torques at the joints while moving the object along its specified trajectory. Furthermore, excessively large values for the internal contact forces may even result in damage or deformity to the object or manipulators. There are two basic approaches to this problem: (i) to explicitly control the internal forces to track reference trajectories or (ii) to calculate the contact forces (including their internal components) by optimization techniques. In the explicit control case, some approaches proposed in the literature require knowledge of dynamics of the manipulators and object (e.g., see $[10,11])$ while others do not (e.g. [9]). Most of the approaches that determine the contact forces to optimize a designer specified criteria involve no servoing and assume knowledge of the dynamics of the held object $[13,17,18,19,20,29]$.

The paper reviews our original approach for dynamic load distribution and explicit position- and internal force-control of the closed chain system consisting of two manipulators securely lifting and transporting a rigid body object in a three dimensional workspace $[29,30$, 33]. A more detailed summary of our past work is described in [32]. The control architecture is dynamic model based, thus the paper will also present a method for deriving a rigid body model for the system. The joint space model given here is a generalization of our previous techniques for modeling the system $[30,31]$. It will be shown that the earlier results are just special cases of the modeling given here.

The paper is organized as follows: A description of the system and the dynamical equations for the manipulators and object are given in section 2. A general framework for load distribution is reviewed in section 3. The kinematic coupling effects are modeled in section 4 and a closed chain dynamical model in the joint space is derived in section 5 . A reduced order model governing the motion of the closed chain and a separate equation for calculating the internal components of the contact forces are the subject of section 6 . A control architecture originally proposed in [31] is reviewed in section 7 where some recent insights into its net effect are discussed. A summary and conclusion are given in the final section.

\section{SYSTEM DESCRIPTION AND DYNAMICS OF MANIPULATORS AND OBJECT}

The system is comprised of two serial link manipulators mutually holding and transporting a rigid body object in a three dimensional workspace. The manipulators and object form a single closed chain mechanism. Manipulator $i(i=1,2)$ has a stationary base and contains $N_{i}$ single DOF joints $\left(N_{i} \geq 6\right.$ in the spatial case). The manipulators can be structurally distinct and possess different capabilities, i.e., they can have an equal $\left(N_{1}=N_{2}\right)$ or unequal $\left(N_{1} \neq N_{2}\right)$ number of joints. The object is rigid and jointless. It assumed that there is no relative motion between the end effectors and object, i.e., the end effectors securely hold the object without any slippage. The configuration of the system is shown in Figure 1.

\subsection{System variables and coordinate frames.}

Let the joint positions, velocities, and accelerations of manipulator $i$ be represented by the $\left(N_{i} \times 1\right)$ vectors $q_{i}=\left[q_{i 1}, q_{i 2}, \ldots, q_{i N_{i}}\right]^{T}, \dot{q}_{i}=\left[\dot{q}_{i 1}, \dot{q}_{i 2}, \ldots, \dot{q}_{i N_{i}}\right]^{T}$, and $\ddot{q}_{i}=$ $\left[\ddot{q}_{i 1}, \ddot{q}_{i 2}, \ldots, \ddot{q}_{i N_{i}}\right]^{T}$, respectively. The joint positions of the two manipulators are the generalized coordinates describing the configuration of the system.

A stationary world coordinate frame $\left(X_{w}, Y_{w}, Z_{w}\right)$ serves as a reference frame. The location of this coordinate frame is based on the task geometry. As shown in Figure 1, the coordinate frame $\left(X_{k}^{(i)}, Y_{k}^{(i)}, Z_{k}^{(i)}\right)$ is assigned to the $k$ th link of manipulator $i$, where $k=1,2, \ldots, N_{i}$. 


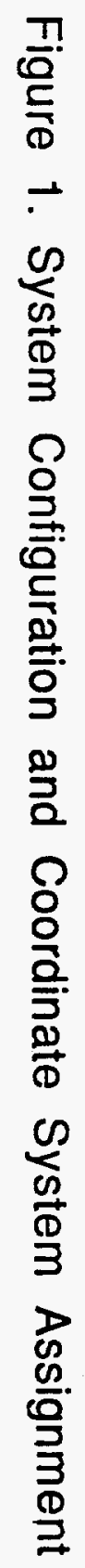
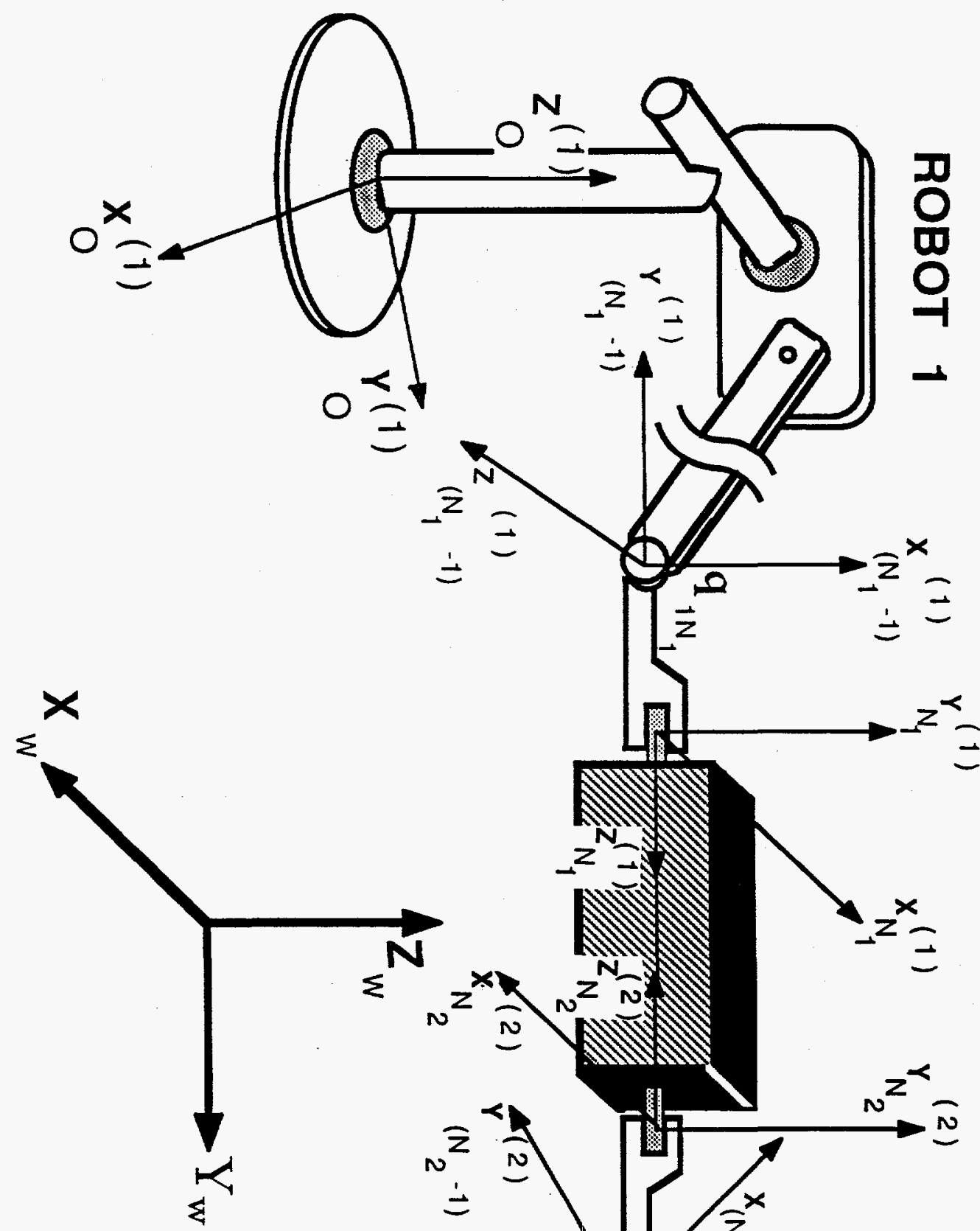

$\circ$ 인

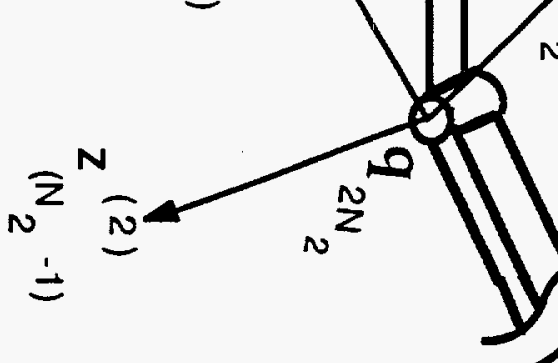




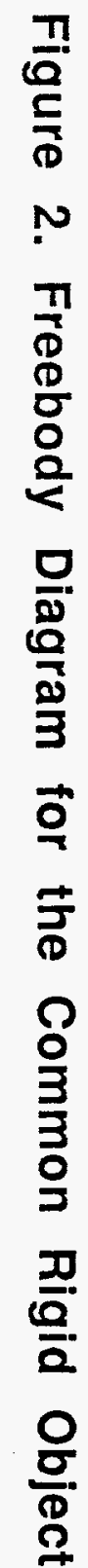

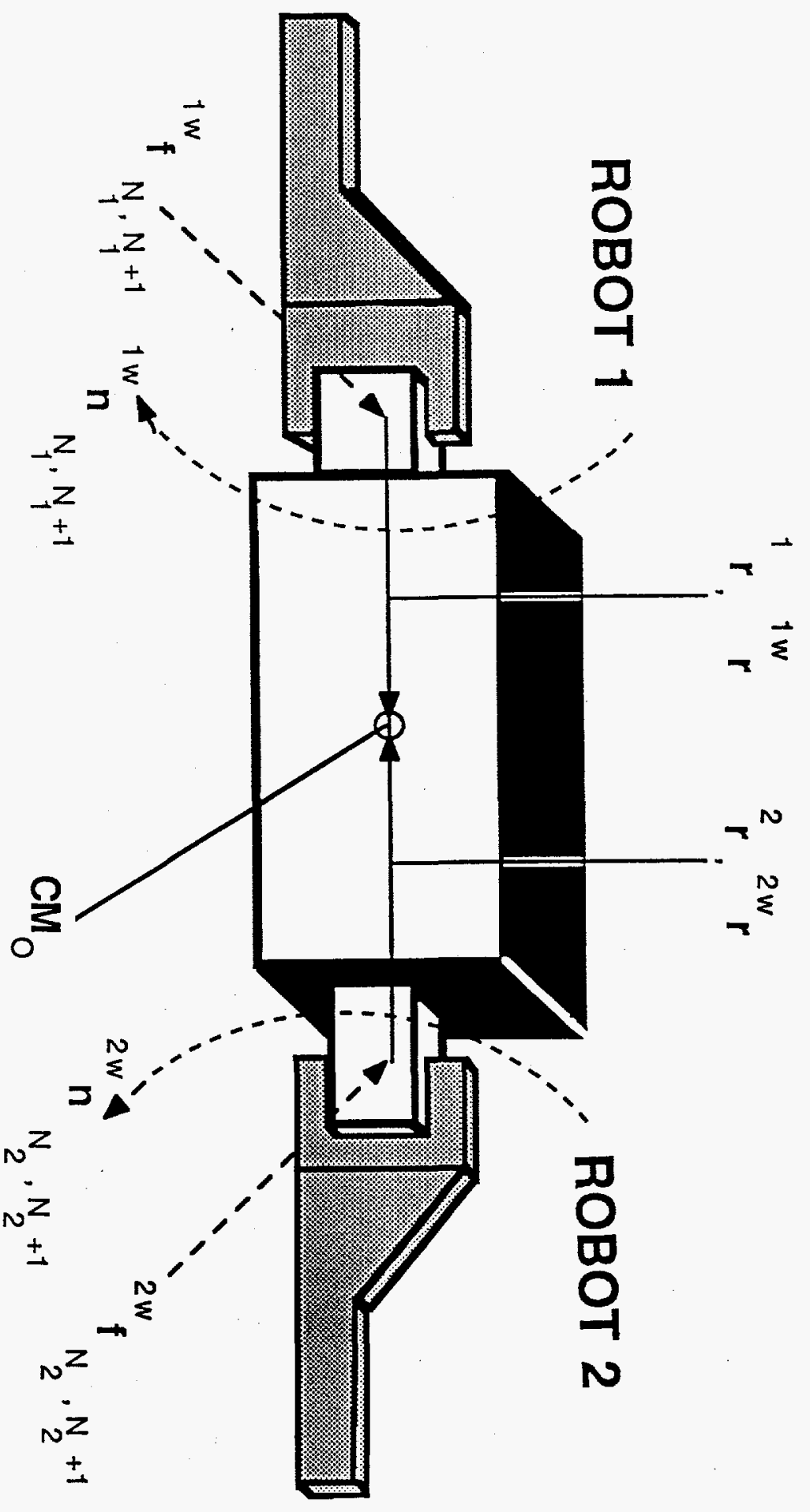


The tips of the $(3 \times 1)$ vectors ${ }^{i} r$ and ${ }^{i w} r$ emanating from the centerpoint of the end effector of manipulator $i$ coincide with the point $C M_{o}$, the center of mass of the rigid object, as shown in Figure 2. ${ }^{i} r$ and ${ }^{i w} r$ are expressed in the end effector and world coordinate frames, respectively. They are related by:

$$
{ }^{i w} r={ }^{i} R_{w}^{N_{i} i} r
$$

where ${ }^{i} R_{w}^{N_{i}}={ }^{i} R_{w}^{N_{i}}\left(q_{i}\right)$ is an orthogonal $(3 \times 3)$ rotation matrix that describes the orientation of the $\left(X_{N_{i}}^{(i)}, Y_{N_{i}}^{(i)}, Z_{N_{i}}^{(i)}\right)$ coordinate frame which has as its origin at the centerpoint of the end effector of manipulator $i$ in the world coordinates.

\subsection{Manipulator Dynamics.}

This section presents the equations of motion of the individual manipulators. The composite dynamics of the manipulators are given by:

$$
\left[\begin{array}{l}
\tau_{1} \\
\tau_{2}
\end{array}\right]=\left[\begin{array}{cc}
D_{1} & 0_{N_{1} \times N_{2}} \\
0_{N_{2} \times N_{1}} & D_{2}
\end{array}\right]\left[\begin{array}{l}
\ddot{q}_{1} \\
\ddot{q}_{2}
\end{array}\right]+\left[\begin{array}{l}
C_{1} \\
C_{2}
\end{array}\right]+\left[\begin{array}{cc}
J_{1 w}^{T} & 0_{N_{1} \times 6} \\
0_{N_{2} \times 6} & J_{2 w}^{T}
\end{array}\right]\left[\begin{array}{l}
f_{c 1} \\
f_{c 2}
\end{array}\right]
$$

where $0_{k \times m}$ denotes a $(k \times m)$ matrix of zeros and superscript $T$ denotes a matrix transpose. The joint torques applied to the joint actuators of manipulator $i$ are signified by the vector $\tau_{i}=\left[\tau_{i 1}, \tau_{i 2}, \ldots, \tau_{i N_{i}}\right]^{T}$. The $\left(N_{i} \times N_{i}\right)$ symmetric, positive definite inertia matrix is $D=D_{i}\left(q_{i}\right)$, and the Coriolis, centripetal, and gravity forces for manipulator $i$ are described by the $\left(N_{i} \times 1\right)$ vector $C_{i}=C_{i}\left(q_{i}, \dot{q}_{i}\right)$.

Each manipulator imparts a contact force ${ }^{i w} f_{N_{i}, N_{i}+1}$ and a contact torque ${ }^{i w} n_{N_{i}, N_{i}+1}$ to the object at and about the centerpoint of the the origin of the end effector for manipulator $i$, respectively, as shown in Figure 2. ${ }^{i w} f_{N_{i}, N_{i}+1}$ and ${ }^{i w} n_{N_{i}, N_{i}+1}$ are expressed in the world coordinates, and the subscript $N_{i}, N_{i}+1$ signifies that the contact force or torque is transmitted from the $N_{i}$ th link of manipulator $i$ to the $\left(N_{i}+1\right)$ th link, where the latter link is the held object itself. The $(6 \times 1)$ vector $f_{c i}$ in eq. (2) signifies the generalized contact force imparted by manipulator $i$. It is defined by:

$$
f_{c i}=\left[\begin{array}{l}
i w \\
{ }^{i} f_{N_{i}, N_{i}+1} \\
{ }^{n_{N_{i}, N_{i}+1}}
\end{array}\right]
$$

In eq. (2), the $\left(N_{i} \times 6\right)$ transposed Jacobian matrix $J_{i w}^{T}=J_{i w}^{T}\left(q_{i}\right)$ transforms the generalized contact force $\neq$ imparted by manipulator $i$ into the joint space. $J_{i w}$ is assumed to possess full rank six.

\subsection{Object Dynamics.}

The dynamics for the rigid object are obtained through application Newton's and Euler's equations of motion. It is convenient to express these equations in a compact form:

$$
Y=L\left[\begin{array}{l}
f_{c 1} \\
f_{c 2}
\end{array}\right]
$$

In eq. (4), $Y$ is a $(6 \times 1)$ vector representing the net force (and torque) acting at the center of mass of the object due to its acceleration and gravity. It is defined by:

$$
Y=\left[\begin{array}{cc}
m_{c} I_{3} & 0_{3 \times 3} \\
0_{3 \times 3} & K_{c}
\end{array}\right]\left[\begin{array}{c}
\dot{v}_{c} \\
\dot{\omega}_{c}
\end{array}\right]+\left[\begin{array}{c}
-m_{c} g \\
\Omega_{c} K_{c} \omega_{c}
\end{array}\right]=\Lambda\left[\begin{array}{c}
\dot{v}_{c} \\
\dot{\omega}_{c}
\end{array}\right]+\left[\begin{array}{c}
-m_{c} g \\
\Omega_{c} K_{c} \omega_{c}
\end{array}\right]
$$

of Generalized contact force will be referred to as contact force hereinafter. 
where $I_{k}$ denotes a $(k \times k)$ identity matrix and where all Cartesian vectors are with respect to the world coordinate system $\left(X_{w}, Y_{w}, Z_{w}\right)$. In eq. (5), $m_{c}$ is the mass of the rigid object, and $K_{c}$ is the $(3 \times 3)$ symmetric inertia matrix of the object about its center of mass. The $(3 \times 1)$ vector $g$ represents the gravitational acceleration of the object. The $(6 \times 1)$ vectors $\left[v_{c}^{T}, \omega_{c}^{T}\right]^{T}$ and $\left[\dot{v}_{c}^{T}, \dot{\omega}_{c}^{T}\right]^{T}$ denote the Cartesian velocity and acceleration of the center of mass of the object, respectively, with $\left(v_{c}, \dot{v}_{c}\right)$ being the translational and $\left(\omega_{c}, \dot{\omega}_{c}\right)$ the rotational components. The $(6 \times 6)$ matrix $\Lambda=\Lambda\left(m_{c}, K_{c}\right)$ is a compact representation of the coefficient matrix of $\left[\dot{v}_{c}^{T}, \dot{\omega}_{c}^{T}\right]^{T}$ in eq. (5) .

In eq. (5) , $\left(\Omega_{\mathrm{c}} K_{\mathrm{c}} \omega_{c}\right)$ is a $(3 \times 1)$ vector arising from expressing the vector cross product expression $\left(\vec{\omega}_{c} \times\left(K_{c} \omega_{c}\right)\right)$ in a matrix-column vector notation, where $\Omega_{c}\left(\omega_{c}\right)$ is a $(3 \times 3)$ skew symmetric matrix [31]:

$$
\Omega=\left[\begin{array}{ccc}
0, & -\omega_{z}, & \omega_{y} \\
\omega_{z}, & 0, & -\omega_{x} \\
-\omega_{y}, & \omega_{x}, & 0
\end{array}\right]
$$

The right side of eq. (4) represents the net force acting on the object at its center of mass due to the contact forces acting at the contact points between the manipulators and object. The $(6 \times 12)$ matrix $L$ in eq. (4) is an explicit function of the $(6 \times 6)$ contact force transmission matrices $L_{1}$ and $L_{2}$ [31]:

$$
L=\left[\begin{array}{ll}
L_{1}, & L_{2}
\end{array}\right]
$$

where matrix $L_{i}(i=1,2)$ is defined by [31]:

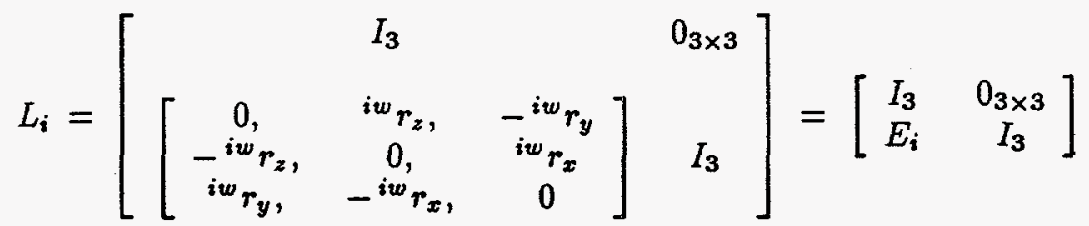

In eq. (8), $E_{i}=E_{i}\left({ }^{i w} r\right)$ is a $(3 \times 3)$ skew symmetric matrix arising from expressing the vector cross product expression $\left(-{ }^{i w} \vec{r} \times{ }^{i w} \vec{f}_{N_{i}, N_{i}+1}\right)$ in a matrix-column vector notation, where $-i w_{r}$ represents a moment arm from point $C M_{o}$ to point of application of $f_{c i}$ (see Figure 2). It should be mentioned that $L_{i}=L_{i}\left(q_{i}\right)$ because ${ }^{i w} r={ }^{i w} r\left(q_{i}\right)$ in accordance with eq. (1) . Interestingly, eq. (8) reveals that $L_{i}$ is nonsingular and that its determinant is equal to one.

In this paper it is assumed that the joint variables of the manipulators in the closed chain configuration are known through feedback of their sensed or measured values or by feedback of their calculated values in a forward dynamic simulation of the system. It is also assumed that the object's mass, inertia, and geometric properties are known, and that a trajectory for the object's center of mass has been specified. Thus matrix $L$ and vector $Y$ in eq. (4) are known quantities.

\section{A GENERAL FRAMEWORK FOR LOAD DISTRIBUTION}

To solve the underspecified dynamic load distribution problem, a new vector variable $\epsilon=$ $\left[\epsilon_{1}, \epsilon_{2}, \ldots, \epsilon_{6}\right]^{T}$ is introduced. Six position controlled degrees of freedom (DOF) are lost due to the closed chain configuration [31]. The number of components of $\epsilon$ is equal to the dimension of the null space of matrix $L$ and reflects the fact that the number of position controlled DOF lost is equal to the number of DOF gained for controlling the internal contact forces [18]. $\epsilon$ is defined by: 


$$
\epsilon=M\left[\begin{array}{l}
f_{c 1} \\
f_{c 2}
\end{array}\right]
$$

The $(6 \times 12)$ matrix $M$ in eq. $(9)$ is selected such that the $(12 \times 12)$ composite matrix $S$, defined by:

$$
S=\left[\begin{array}{c}
L \\
M
\end{array}\right]
$$

is nonsingular.

It is convenient to partition the inverse of $S$ into two matrices:

$$
S^{-1}=\left[\begin{array}{ll}
\Phi, & \Psi
\end{array}\right]
$$

where $\Phi$ and $\Psi$ are $(12 \times 6)$ matrices. Eqs. (10) and (11) imply five matrix identities:

$$
L \Phi=I_{6}, L \Psi=0_{6 \times 6}, \quad M \Phi=0_{6 \times 6}, M \Psi=I_{6}, \quad \Phi L+\Psi M=I_{12}
$$

where, here again, $I_{k}$ and $0_{k \times l}$ denote a $(k \times k)$ identity matrix and a $(k \times l)$ matrix of zeros, respectively.

The identity $L \Psi=0_{6 \times 6}$ reveals that the column vectors comprising $\Psi$ lie in and span the null space of $L$. Observing eq. (7), an obvious choice for $\Psi$ is:

$$
\Psi=\left[\begin{array}{c}
-L_{1}^{-1} \\
L_{2}^{-1}
\end{array}\right]
$$

Matrix $\Psi$ is not unique. Indeed, postmultiplying the choice for $\Psi$ in eq. (13) by an arbitrary $(6 \times 6)$ nonsingular matrix yields a new $\Psi$ which lies in the null space of $L$. In this paper it is assumed that $\{L, \Psi\}$ are known quantities. The designer chooses $M$ to satisfy $M \Psi=I_{6}$. Then, given $\{L, \Psi, M\}, \Phi$ is determined based on the matrix identities in eq. (12). These issues will be discussed in later in this section.

Eqs. (4) and (9) can be solved for the contact forces [29, 30]:

$$
\left[\begin{array}{l}
f_{c 1} \\
f_{c 2}
\end{array}\right]=\Phi Y+\Psi \epsilon
$$

in which eq. (11) has been invoked. The second term $\{\Psi \epsilon\}$ on the right of eq. (14) is the homogeneous solution to eq. (4) and is a component of $\left[f_{c 1}^{T}, f_{c 2}^{T}\right]^{T}$ which causes internal stress and torsion in the object but does not contribute to its motion since $L \Psi \epsilon=0_{6 \times 1}$. The first term $\{\Phi Y\}$ on the right of eq. (14) is a particular solution to eq. (4) and is the component of $\left[f_{c 1}^{T}, f_{c 2}^{T}\right]^{T}$. which causes the object to physically move, since $L \Phi Y=Y$. However, it will be shown in this paper that the particular solution to eq. (4) can contain a component which lies in the null space of $L$, and such a component causes internal stress and torsion in the object but does not contribute to its motion. This has been demonstrated previously in a dual manipulator context in [19] by a different approach which studied the characteristics of a class of pseudoinverses of $L$, but the approach given here is conceptually simpler.

The symbolic solution for the contact forces given by eq. (14) is significant because it indicates that the designer can specify the distribution of the payload's mass between the two manipulators by the choice of $M$ and $\epsilon$. For example, since $Y$ is known, matrix $\Phi$ governs the distribution of the payload among the motion inducing components in the contact force solution. 
3.1. Identifying Motion Inducing and Internal Stress Components of $(\Phi Y)$. Any vector in the 12-dimensional linear space describing the contact forces imparted to the object by the manipulators can be expressed as linear combinations of two orthogonal subspaces: the exact range space of $L^{T}$ and the null space $\Psi$ of $L$. It is convenient to introduce the basis $V$ :

$$
V=\left[\begin{array}{ll}
L^{T}, & \Psi
\end{array}\right]
$$

It is easy to see that the columns vectors comprising $V$ span the 12-dimensional linear space. Matrix $\Phi$ can be expressed in terms of $V$ :

$$
\Phi=L^{T} \alpha+\Psi \gamma
$$

where $\alpha$ and $\gamma$ are $(6 \times 6)$ parameter matrices, respectively. It is easy to verify that $\alpha=$ $\left(L L^{T}\right)^{-1}$ and $\gamma=-M L^{T}\left(L L^{T}\right)^{-1}$ by premultiplying eq. (16) by $L$ and $M$, respectively, and noting eq. (12). Substituting the solutions for $\{\alpha, \gamma\}$ into eq. (16) yields [29]:

$$
\Phi=L^{T}\left(L L^{T}\right)^{-1}-\Psi M L^{T}\left(L L^{T}\right)^{-1}
$$

Eq. (17) reveals that $(\Phi Y)$ always contains a component $\left\{L^{T}\left(L L^{T}\right)^{-1} Y\right\}$ which contributes to the object's motion, but it may also contain a component $\left\{-\Psi M L^{T}\left(L L^{T}\right)^{-1} Y\right\}$ which induces internal stress and torsion in the object in the general case.

It is insightful to substitute for $\Phi$ in eq. (14) using eq. (17) :

$$
\left[\begin{array}{l}
f_{c 1} \\
f_{c 2}
\end{array}\right]=L^{T}\left(L L^{T}\right)^{-1} Y-\Psi\left(M L^{T}\left(L L^{T}\right)^{-1} Y-\epsilon\right)
$$

Eq. (18) describes all possible solutions to eq. (4) in terms of the basis $V$. Each solution in the family is distinguished by the designer's choice for the quantities $\{\Psi, M, \epsilon\}$. Interestingly, each and every distinct solution in the family has the identical object motion inducing component. Therefore the difference between any two distinct solutions lies in the null space of $L$.

\subsection{Choosing Matrix $M$}

The purpose of this section is determine a family of solutions for $M$ which results in $S$ being nonsingular and satisfies $M \Psi=I_{6}$ when $\Psi$ is known. We then present three possible choices for $M$ and calculate $\Phi$ for each of the choices. It is also shown how each choice for $M$ can be obtained by selecting a parameter matrix in the family of solutions for $M$.

$M$ can be expressed in terms of the basis $V$ defined in eq. (15) :

$$
M=\beta L+\zeta \Psi^{T}
$$

where $\beta$ and $\zeta$ are $(6 \times 6)$ parameter matrices. It is easy to verify that $\zeta=\left(\Psi^{T} \Psi\right)^{-1}$ by postmultiplying eq. (19) by $\Psi$ and observing eq. (12). Substituting the solution for $\zeta$ in eq. (19) obtains:

$$
M=\beta L+\left(\Psi^{T} \Psi\right)^{-1} \Psi^{T}
$$

When $M$ is defined by eq. (20), $M^{T}$ will always contain a component that lies in the null space of $L$ and therefore $S$ will be nonsingular. Indeed, eq. (20) describes a family of solutions for $M$, and the each distinct member of the family is characterized by the designer's choice for $\beta$.

Example 1. Choosing $M$ to Obtain a Previous Result

The dynamic load distribution problem that arises when two manipulators mutually lift a rigid object was not discussed in our earlier work [31] that modeled the closed chain configuration shown in Figure 1. The approach in [31] to modeling the dynamic coupling 
effects between the manipulators was to make the contact forces imparted by manipulator 1 implicit variables using the following procedure: (i) solve eq. (4) for $f_{c 1}\left[=L_{1}^{-1}\left(Y-L_{2} f_{c 2}\right)\right]$ (ii) substitute for $f_{c 1}$ into eq. (2) using its solution obtained in step i. The resulting equation represents the composite dynamics of both manipulators and the object and is an explicit function of $f_{c 2}$. The physical interpretation of this modeling procedure was not discussed in [31].

In this example it is shown that the result of [31] can be obtained by an application of the general load distribution procedure presented here. The modeling procedure in [31] is obtained by selecting matrices $\Psi$ and $M$ to be:

$$
\begin{aligned}
\Psi & =\left[\begin{array}{cc}
-L_{1}^{-1} & L_{2} \\
I_{6}
\end{array}\right] \\
M & =\left[\begin{array}{ll}
0_{6 \times 6}, & I_{6}
\end{array}\right]
\end{aligned}
$$

It should be noted that eq. (21) is obtained by postmultiplying the choice for $\Psi$ in eq. (13) by $L_{2}$. Further, the choice for $M$ in eq. (22) is obtained from eq. (20) by selecting $\beta$ to be:

$$
\beta=\left(\Psi^{T} \Psi\right)^{-1} L_{2}^{T}\left(L_{1} L_{1}^{T}\right)^{-1}
$$

Substituting eqs. (21) and (22) into eq. (17) yields the solution for $\Phi$ :

$$
\Phi=\left[\begin{array}{c}
L_{1}^{-1} \\
0_{6 \times 6}
\end{array}\right]
$$

Substituting for $\{\Psi, \Phi\}$ in eq. (14) using eqs. (21) and (24) and inserting the result into eq. (2) yields the model in [31] where $\epsilon=f_{c 2}$. The procedure in [31] has unknowingly distributed the load such that only manipulator 1 induces the object to physically move in space whereas the contact forces imparted by manipulator 2 are purely internal. In this extreme case, manipulator 1 bears the entire load.

Example 2. Choosing $M$ to be a Function of Constrained Parameters.

Here $\Psi$ is defined by eq. (13). In this example matrix $M$ is selected to be a function of the force transmission matrices $\left\{L_{1}, L_{2}\right\}$ and two unknown scalar parameters $\left\{c_{1}, c_{2}\right\}$ whose values are restricted as follows [29, 30]:

$$
c_{1}+c_{2}=1
$$

Suppose $M$ is chosen to be $[29,30]$ :

$$
M=\left[-c_{2} L_{1}, \quad c_{1} L_{2}\right]
$$

which is obtained from eq. (20) by selecting $\beta$ to be:

$$
\beta=c_{1} I_{6}-\left(\Psi^{T} \Psi\right)^{-1}\left(L_{2} L_{2}^{T}\right)^{-1}
$$

The symbolic solution for $\Phi$ can be determined by substituting for $\Psi$ and $M$ in eq. (17) using eqs. (13) and (26), respectively, and simplifying:

$$
\Phi=\left[\begin{array}{ll}
c_{1} & L_{1}^{-1} \\
c_{2} & L_{2}^{-1}
\end{array}\right]
$$

Since $\left\{c_{1}, c_{2}\right\}$ are variables, $(\Phi Y)$ constitutes a family of particular solutions to eq. (4) when $\Phi$ is defined by eq. (28). It is repeated for emphasis that only the internal component of the particular solution ( $\Phi Y$ ) to eq. (4) is a function of $M$. Therefore the terms in eq. (18) that are explicit functions of $\left\{c_{1}, c_{2}\right\}$ only affect the internal stress and torsion in the held object when eq. (26) applies. 
Example 3. Choosing $M$ So That $M^{T}$ Lies in the Null Space of $L$

Here again $\Psi$ is defined by eq. (13). Suppose that $M$ is selected to be [29]:

$$
M=\left(\Psi^{T} \Psi\right)^{-1} \Psi^{T}=\left[\left(L_{1} L_{1}^{T}\right)^{-1}+\left(L_{2} L_{2}^{T}\right)^{-1}\right]^{-1}\left[-\left(L_{1}^{T}\right)^{-1},\left(L_{2}^{T}\right)^{-1}\right]
$$

It is easy to see that eq. (29) is just a special case of eq. (20) with $\beta$ selected as:

$$
\beta=0_{6 \times 6}
$$

When eq. (29) applies, $M^{T}$ lies in the null space of $L$, i.e., $L M^{T}=0_{6 \times 6}$ and eq. (17) immediately simplifies:

$$
\Phi=L^{T}\left(L L^{T}\right)^{-1}=\left[\begin{array}{l}
L_{1}^{T} \\
L_{2}^{T}
\end{array}\right]\left(L_{1} L_{1}^{T}+L_{2} L_{2}^{T}\right)^{-1}
$$

where eq. (7) has been applied. Since the internal force component of $(\Phi Y)$ has vanished, the terms $(\Phi Y)$ and $(\Psi \epsilon)$ in eq. (14) are now mutually orthogonal because:

$$
\Phi^{T} \Psi=0_{6 \times 6} \text {. }
$$

and orthogonality is the strongest form of linear independence between a pair of vectors [36].

The modeling of the kinematic coupling effects occurring between the manipulators is discussed next.

\section{MODELING OF KINEMATIC COUPLING EFFECTS}

There are two purposes for this section. First, a linear transformation relating the Cartesian velocity vector of the object and the vector of joint velocities for both manipulators will be derived. This relationship will be useful for expressing the object's dynamical equations in the joint space. Second, a set of rigid body kinematic constraints which must be satisfied by the joint velocities of the manipulators will be derived.

A linear relationship between the Cartesian velocity of the object at point $C M_{0}$ and at the point of application of the contact force imparted by manipulator $i$, i.e., the centerpoint of the end effector, is established using the theory of infinitesimal rotation of a rigid object $[37,31]$ :

$$
\left[\begin{array}{c}
v_{i} \\
\omega_{i}
\end{array}\right]=L_{i}^{T}\left[\begin{array}{c}
v_{c} \\
\omega_{c}
\end{array}\right]
$$

where the $(3 \times 1)$ vectors $v_{i}$ and $\omega_{i}$ represent the Cartesian translational and rotational velocities, respectively, of the end effector of manipulator $i$ in the world coordinates.

Substituting for $L_{i}^{T}$ in eq. (33) using eq. (8) verifies that $\omega_{i}=\omega_{c}$ as expected. Indeed, the Cartesian angular velocities of the end effectors and object are identical due to the assumption that the manipulators securely hold the object without any slippage.

Combining the two sets of equations obtained from eq. (33) with $i=1,2$ gives:

$$
\left[\begin{array}{c}
v_{1} \\
\omega_{1} \\
v_{2} \\
\omega_{2}
\end{array}\right]=\left[\begin{array}{c}
L_{1}^{T} \\
L_{2}^{T}
\end{array}\right]\left[\begin{array}{l}
v_{c} \\
\omega_{c}
\end{array}\right]=L^{T}\left[\begin{array}{c}
v_{c} \\
\omega_{c}
\end{array}\right]
$$

There is a well specified solution for the object velocities $\left[v_{c}^{T}, \omega_{c}^{T}\right]^{T}$ based on eq. (34) because $L$ has full rank six and $\left[v_{i}^{T}, \omega_{i}^{T}\right]^{T}$ lies in the exact range space of $L_{i}^{T}$. The solution is obtained by premultiplying eq. (34) by matrix $\Phi^{T}$ and noting eq. (12) : 


$$
\left[\begin{array}{c}
v_{c} \\
\omega_{c}
\end{array}\right]=\Phi^{T}\left[\begin{array}{l}
v_{1} \\
\omega_{1} \\
v_{2} \\
\omega_{2}
\end{array}\right]
$$

Three distinct solutions for $\Phi$ were obtained in the three examples of Section 3.2 given choices for $\Psi$ and $M$. It is straightforward to verify that substituting for $\Phi^{T}$ in eq. (35) using each of the three solutions (for $\Phi$ ) and applying eq. (33) yields $\left[v_{c}^{T}, \omega_{c}^{T}\right]^{T}=\left[v_{c}^{T}, \omega_{c}^{T}\right]^{T}$.

The velocities of the end effector of manipulator $i$ in the Cartesian world coordinate frame and the joint space are related through the $\left(6 \times N_{i}\right)$ Jacobian matrix $J_{i w}$, i.e.:

$$
\left[\begin{array}{c}
v_{i} \\
\omega_{i}
\end{array}\right]=J_{i w} \dot{q}_{i}
$$

Substituting for $\left[v_{i}^{T}, \omega_{i}^{T}\right]^{T}$ in eq. (35) using eq. (36) with $i=1,2$ relates the Cartesian velocities of the object at its center of mass to the joint space:

$$
\left[\begin{array}{c}
v_{c} \\
\omega_{c}
\end{array}\right]=\Phi^{T}\left[\begin{array}{cc}
J_{1 w} & 0_{6 \times N_{2}} \\
0_{6 \times N_{2}} & J_{2 w}
\end{array}\right]\left[\begin{array}{l}
\dot{q}_{1} \\
\dot{q}_{2}
\end{array}\right]=\Phi^{T} J\left[\begin{array}{l}
\dot{q}_{1} \\
\dot{q}_{2}
\end{array}\right]
$$

The $\left(12 \times\left(N_{1}+N_{2}\right)\right)$ composite Jacobian matrix $J=J\left(q_{1}, q_{2}\right)$ in eq. (37) has full rank twelve since the $J_{i w}$ has full rank six.

It is easy to see from Figure 1 that the end effectors of the manipulators cannot move independently when they mutually hold the rigid body object. The constraint between the Cartesian velocities of the end effectors is obtained by premultiplying eq. (34) by $\Psi^{T}$ and noting eq. (12) :

$$
\Psi^{T}\left[\begin{array}{c}
v_{1} \\
\omega_{1} \\
v_{2} \\
\omega_{2}
\end{array}\right]=0_{6 \times 1}
$$

The constraint can be expressed in the joint space by substituting for $\left[v_{i}^{T}, \omega_{i}^{T}\right]^{T}$ in eq. (35) using eq. (36) with $i=1,2$ :

$$
\Psi^{T} J\left[\begin{array}{c}
\dot{q}_{1} \\
\dot{q}_{2}
\end{array}\right]=A\left[\begin{array}{c}
\dot{q}_{1} \\
\dot{q}_{2}
\end{array}\right]=0_{6 \times 1}
$$

where the $\left(6 \times\left(N_{1}+N_{2}\right)\right)$ matrix $A=A\left(q_{1}, q_{2}\right)\left(=\Psi^{T} J\right)$ is assumed to have full rank six.

Let ${ }^{k} J$ denote the $k$ th column vector of $J,\left(k=1,2, \ldots, N_{1}+N_{2}\right)$. Since ${ }^{k} J$ is a twelve dimensional vector, it can be expressed in terms of the basis $V$ defined in eq. (15) :

$$
{ }^{k} J=L^{T} \alpha+\Psi \gamma
$$

where $\alpha$ and $\gamma$ are $(6 \times 1)$ parameter vectors. If $\gamma=0_{6 \times 1}$ then the $k$ th column of $A\left(=\Psi^{T k} J\right)=0_{6 \times 1}$ because $L \Psi=0_{6 \times 6}$. In this case, none of the kinematic constraints in eq. (39) would be a function of the $k$ th element of the vector of joint velocities $\left[q_{1}^{T}, q_{2}^{T}\right]^{T}$. Therefore it is further assumed that each column vector comprising $J$ has a nonzero component lying in the null space of $L$.

Eq. (39) comprises six scalar constraint equations characterizing the kinematic dependence among the joint velocities when the manipulators operate in the closed chain configuration. Each independent scalar constraint contained in eq. (39) causes the loss of one DOF in the closed chain [37]. Indeed, the DOF of the entire closed chain system is $\left(N_{1}+N_{2}-6\right)$. 

next.

A dynamical model for the multiple manipulator system in the joint space is presented

\section{DERIVATION OF RIGID BODY MODEL IN JOINT SPACE}

The two manipulators and object form a single closed chain mechanism, and a rigid body model governing the motion of the closed chain is derived in the joint space in this section. In the ensuing development it is useful to define $N_{12}=N_{1}+N_{2}$. eq. (14) :

The first step in deriving this model is to substitute for $\left[f_{c 1}^{T}, f_{c 2}^{T}\right]^{T}$ in eq. (2) using

$$
\tau=\left[\begin{array}{cc}
D_{1} & 0_{N_{1} \times N_{2}} \\
0_{N_{2} \times N_{1}} & D_{2}
\end{array}\right] \ddot{q}+\left[\begin{array}{c}
C_{1} \\
C_{2}
\end{array}\right]+J^{T} \Phi Y+A^{T} \epsilon
$$

where $J$ is defined in conjunction with eq. (37) and where $q=\left[q_{1}^{T}, q_{2}^{T}\right]^{T}, \dot{q}=\left[\dot{q}_{1}^{T}, \dot{q}_{2}^{T}\right]^{T}$, $\ddot{q}=\left[\ddot{q}_{1}^{T}, \ddot{q}_{2}^{T}\right]^{T}$, and $\tau=\left[\tau_{1}^{T}, \tau_{2}^{T}\right]^{T}$. Interestingly, it is observed that the coefficient matrix of $\epsilon$ in eq. (41) is just the transpose of the coefficient matrix of the vector of joint velocities in the kinematic constraints given by eq. (39) .

Vector $Y$ in eq. (41) is a function of the Cartesian space variables $\left\{\omega_{c}, \dot{v}_{c}, \dot{\omega}_{c}\right\}$ according to its definition in eq. (5) . $Y$ can be expressed in the joint space by substituting for $\omega_{c}$ and $\left[\dot{v}_{c}^{T}, \dot{\omega}_{c}^{T}\right]^{T}$ in eq. (5) using eq. (37) and its time derivative, respectively:

$$
Y=\Lambda \Phi^{T} J \ddot{q}+\Lambda\left(\Phi^{T} \dot{J}+\dot{\Phi^{T}} J\right) \dot{q}+\left[\begin{array}{c}
-m_{c} g \\
\Omega_{c} K_{c}\left[\begin{array}{c}
0 \times 3 \\
0,
\end{array} I_{3}\right] \Phi^{T} J \dot{q}
\end{array}\right]
$$

In eq. (42), the $(12 \times 6)$ and $\left(12 \times N_{12}\right)$ matrices $\dot{\Phi}[=(d \Phi / d t)]$ and $j[=(\partial J / \partial q) \dot{q}]$, respectively, are both functions of the variables $\{q, q\}$. The occurrence of $\omega$ on the right of eq. (5) has been replaced by $\left[0_{3 \times 3}, I_{3}\right] \Phi^{T} J \dot{q}$ in eq. (42). The components $\left\{\omega_{x}, \omega_{y}, \omega_{z}\right\}$ in matrix $\Omega$ are expressed in the joint space using this transformation, so $\Omega=\Omega(q, \dot{q})$ in eq. (42) .

Substituting for $Y$ in eq. (41) using eq. (42) and rearranging terms yield the closed chain dynamics in the joint space:

$$
\tau=D \ddot{q}+C+H_{m} \dot{q}+H_{v}+A^{T} \epsilon
$$

The $\left(N_{12} \times N_{12}\right)$ matrix $D=D(q)$ in eq. (43) is the inertia matrix for the entire system. It is defined by:

$$
D=\left[\begin{array}{cc}
D_{1} & 0_{N_{1} \times N_{2}} \\
0_{N_{2} \times N_{1}} & D_{2}
\end{array}\right]+J^{T} \Phi \Lambda \Phi^{T} J
$$

Since $D_{i}$ is positive definite, the first term to the right of eq. (44) is positive definite. The second term to the right of eq. (44) is positive semidefinite. Therefore $D$ is positive definite because the sum of a positive definite matrix and a positive semidefinite matrix is positive definite [36].

The $\left(N_{12} \times 1\right)$ vector $C=C(q, \dot{q})$ is defined by:

$$
C=\left[\begin{array}{l}
C_{1} \\
C_{2}
\end{array}\right]
$$

The $\left(N_{12} \times N_{12}\right)$ matrix $H_{m}=H_{m}(q, \dot{q})$ and the $\left(N_{12} \times 1\right)$ vector $H_{v}=H_{v}(q, \dot{q})$ in eq. (43) are defined by:

$$
H_{m}=J^{T} \Phi \Lambda\left(\Phi^{T} \dot{J}+\dot{\Phi}^{T} J\right)
$$




$$
H_{v}=J^{T} \Phi\left[\begin{array}{c}
-m_{c} g \\
\Omega_{c} K_{c}\left[\begin{array}{c}
0 \times 3 \\
0_{3}
\end{array}\right] \Phi^{T} J \dot{q}
\end{array}\right]
$$

It should be mentioned that the closed chain dynamical model derived in [30] is just a special case of eq. (43) with $\{\Psi, \Phi\}$ defined by eqs. (13) and (28), respectively.

Eq. (43) accounts for the dynamics of all components of the closed chain but does not satisfy the rigid body kinematic constraints in eq. (39). Indeed, eq. (43), along with the time derivative of eq. (39) :

$$
A \ddot{q}+\dot{A} \dot{q}=0_{6 \times 1}
$$

govern the motion of the closed chain dual manipulator system and the internal component of the contact forces. The $\left(6 \times N_{12}\right)$ matrix $\dot{A}[=(\partial A / \partial q) \dot{q}]$ in eq. (48) is a function of the variables $\{q, \dot{q}\}$.

The form of eqs. (43) and (48) has been obtained for a broad class of constrained rigid body mechanical systems in $[38,39]$ using the method of Lagrange undetermined multipliers [37]. However, it is very unclear how the issues of dynamically distributing the load and relating $\epsilon$ to internal contact forces would be addressed if the modeling techniques given in $[38,39]$ were applied to the multiple manipulator closed chain considered here.

The $\left(N_{12}+6\right)$ scalar equations comprising eqs. (43) and (48) can be used to accomplish a forward dynamics simulation of the system where the quantities $\{\ddot{q}, \epsilon\}$ are unknowns when the joint torques $\tau$ are specified. A symbolic solution for $\{\vec{q}, \epsilon\}$ based on eqs. (43) and (48) can be obtained by inverting the coefficient matrix of $\left[\ddot{q}^{T}, \epsilon^{T}\right]^{T}$ using inverse by partitioning [36]:

$$
\begin{gathered}
\ddot{q}=D^{-1} \Delta\left(\tau-C-H_{m} \dot{q}-H_{v}\right)-D^{-1} A^{T}\left(A D^{-1} A^{T}\right)^{-1} \dot{A} \dot{q} \\
\epsilon=\left(A D^{-1} A^{T}\right)^{-1}\left\{A D^{-1}\left(\tau-C-H_{m} \dot{q}-H_{v}\right)+\dot{A} \dot{q}\right\}
\end{gathered}
$$

The solution for $\epsilon$ in eq. (50) is based on the invertibility of the quantity $\left(A D^{-1} A^{T}\right) . D^{-1}$ is positive definite because $D$ is. Given that $A$ has full rank six, $\left(A D^{-1} A^{T}\right)$ is positive definite and therefore nonsingular. In eq. (49), $\Delta$ is a $\left(N_{12} \times N_{12}\right)$ matrix defined by:

$$
\Delta=I_{N_{12}}-A^{T}\left(A D^{-1} A^{T}\right)^{-1} A D^{-1}
$$

where, here again, $N_{12}=N_{1}+N_{2}$ and $I_{k}$ signifies an $(k \times k)$ identity matrix. By a mathematical observation, $\Delta$ is idempotent, i.e., $\Delta^{2}=\Delta$, and therefore singular, since the only nonsingular idempotent matrix is the identity matrix [36]. It has been shown in our earlier work [31] that the rank of $\Delta$ equals the number of degrees of freedom in the closed chain, i.e., $\operatorname{rank}\{\Delta\}=N_{12}-6$.

While the joint space model is useful for understanding how the system evolves with time in response to applied joint torque inputs, it is not useful for the controller design process. Indeed, the number of scalar equations in eqs. (43) and (48) (or in eqs. (49) and (50), which may also be viewed as a rigid body model) exceed the number of joint torque inputs. However, it is important to note that there is a well specified solution for $\tau$ based on the rigid body model. Since the rank of $\Delta$ equals $\left(N_{12}-6\right)$ and $D$ is positive definite, the rank of the coefficient matrix $\left(D^{-1} \Delta\right)$ of $\tau$ in eq. (49) is also equal to $\left(N_{12}-6\right)$ [40]. Therefore an additional six independent scalar equations that are linear functions of $\tau$ are needed to yield a well specified solution for the $N_{12}$ joint torques $\tau$. The six equations are provided by eq. (50) . Rather than attempting to design a model based controller by solving eqs. (49) and (50) (or eqs. (43) and (48) ) for the joint torques, we will derive a reduced order model and design a control architecture based on it. This is discussed next. 


\section{REDUCED ORDER MODEL}

The joint velocities and accelerations form coupled sets of generalized velocities and accelerations for describing the configuration of the closed chain system, respectively. Linear transformations which express these variables in terms of new independent generalized velocities and accelerations are derived and then applied to eliminate $\{\dot{q}, \ddot{q}\}$ from the closed chain model given by eq. (43) in this section. Then, building on the seminal work in [38], linear transformations are applied to the closed chain dynamics in eq. (43) to separate the model into two sets of equations. The sets of equations govern the motion of the closed chain and the behavior of the internal component of the contact forces, respectively.

A new vector variable $\nu=\left[\nu_{1}, \nu_{2}, \ldots, \nu_{N_{12}}\right]^{T}$ referred to as the pseudovelocity vector $[41,42,39]$ is introduced. The pseudovelocity vector is defined by:

$$
\nu=B \dot{q}
$$

where the $\left(\left(N_{12}-6\right) \times N_{12}\right)$ matrix $B=B(q)$ selected so that the composite $\left(N_{12} \times N_{12}\right)$ matrix $U$, defined by:

$$
U=\left[\begin{array}{l}
A \\
B
\end{array}\right]
$$

is nonsingular, where here again, $A$ is defined in conjunction with eq. (39) and $N_{12}=$ $N_{1}+N_{2}$.

It is convenient to partition the inverse of $U$ into two matrices:

$$
U^{-1}=[\Upsilon, \Gamma]
$$

where $\Upsilon=\Upsilon(q)$ is an $\left(N_{12} \times 6\right)$ matrix and $\Gamma=\Gamma(q)$ an $\left(N_{12} \times\left(N_{12}-6\right)\right)$ matrix. Eqs. (53) and (54) imply five matrix identities:

$A \Upsilon=I_{6}, A \Gamma=0_{6 \times\left(N_{12}-6\right)}, M \Upsilon=0_{\left(N_{12}-6\right) \times 6}, M \Gamma=I_{N_{12}-6}, \Upsilon A+\Gamma B=I_{N_{12}}$

The identity $A \Gamma=0_{6 \times\left(N_{12}-6\right)}$ reveals that the column vectors comprising $\Gamma$ lie in and span the null space of $A$. $\Gamma$ can be determined by the following procedure. Noting that $A=\Psi^{T} J$ and $L \Psi=0_{6 \times 6}$, six vectors lying in the null space (of $A$ ) are given by:

$$
J^{T}\left(J J^{T}\right)^{-1} L^{T}
$$

If $N_{1}=N_{2}=6$, then the above set of vectors spans the null space and is assigned to $\Gamma$. If one or both of the manipulators is kinematically redundant, then $\left(N_{12}-12\right)$ additional vectors are needed to span the null space. By a mathematical observation, $\left(N_{12}-12\right)$ is the dimension of the null space of $J$, and any vector lying in the null space of $J$ also lies in the null space of $A$. The null space of $J$ can be determined by the zero eigenvalue matrix theorem [43].

All vectors lying in the $N_{12}$-dimensional articular space may be expressed in terms of the following basis $Z$ :

$$
Z=\left[\begin{array}{ll}
A^{T}, & \Gamma
\end{array}\right]
$$

It is straightforward to verify that $\Upsilon$ can be expressed in terms of this basis:

$$
\Upsilon=A^{T}\left(A A^{T}\right)^{-1}-\Gamma B A^{T}\left(A A^{T}\right)^{-1}
$$

Eqs. (39) and (52) can be solved for the generalized velocities $\dot{q}$ :

$$
\dot{q}=\Gamma \nu
$$


Differentiating eq. (52) with respect to time establishes the linear relationship between the pseudoaccelerations and the joint accelerations:

$$
\dot{\nu}=B \ddot{q}+\dot{B} \dot{q} .
$$

The $\left(\left(N_{12}-6\right) \times N_{12}\right)$ matrix $\dot{B}[=(\partial B / \partial q) \dot{q}]$ in eq. (59) is a function of the variables $\{q, \dot{q}\}$. Eqs. (48) and (59) can be solved for $\ddot{q}$ :

$$
\ddot{q}=\Gamma \dot{\nu}-[\Upsilon \dot{A}+\Gamma \dot{B}] \Gamma \nu
$$

where eq. (58) has been used. A solution for $\ddot{q}$ may also be obtained by differentiating eq. (58) with respect to time:

$$
\ddot{q}=\Gamma \dot{\nu}+\dot{\Gamma} \nu
$$

where the $\left(N_{12} \times\left(N_{12}-6\right)\right)$ matrix $\dot{\Gamma}[=(\partial \Gamma / \partial q) \dot{q}]$ is a function of the variables $\{q, \dot{q}\}$. identity:

Eqs. (60) and (61) are mathematically equivalent because of the following matrix

$$
\dot{\Gamma}=-[\Upsilon \dot{A}+\Gamma \dot{B}] \Gamma
$$

Eq. (62) is obtained by differentiating the identity: $\Upsilon A+\Gamma B=I_{N_{12}}$ with respect to time and postmultiplying the resulting equation by $\Gamma$.

Substituting for $\dot{q}$ in eq. (39) using eq. (58) yields the kinematic constraint equation $A \Gamma \nu=0_{6 \times 1}$, which is identically true since $A \Gamma=0_{6 \times N_{12}-6}$. Therefore, the kinematic constraints at the velocity level are satisfied regardless of the values of the pseudovelocities when eq. (58) applies. Likewise, substituting for $\{\dot{q}, \ddot{q}\}$ in eq. (48) using eqs. (58) and (61) reveals that the kinematic constraints at the acceleration level are also satisfied regardless of the values of $\{\nu, \dot{\nu}\}$. These findings lead to the observation that expressing the closed chain dynamical model given by eqs. (43) and (48) in terms of the pseudovariables results in the eq. (43) alone representing a rigid body model of the multiple manipulator system:

$$
D \Gamma \dot{\nu}=\tau-C-H_{v}-A^{T} \epsilon+\left(D[\Upsilon \dot{A}+\Gamma \dot{B}]-H_{m}\right) \Gamma \nu
$$

The number of equations in eq. (63) equals the sum of the position controlled DOF and the internal force controlled DOF in the closed chain system.

It is important to note that eq. (63) is still a nonlinear function of the joint positions $q$, i.e., $D=D(q), C=C(q, \nu), H_{m}=H_{m}(q, \nu)$, and $H_{v}=H_{v}(q, \nu)$. Thus it is difficult to perform a forward dynamics simulation of the system based on eq. (63). However, as will now be shown, performing a linear transformation on eq. (63) makes the resulting set of equations valuable for controller design purposes.

Premultiplying eq. (63) by the nonsingular matrix $\left[\Gamma, D^{-1} A^{T}\right]^{T}$ and utilizing eq. (54) separates the model into two sets of equations governing the position controlled DOF and the internal force controlled DOF, respectively:

$$
\begin{gathered}
\Gamma^{T} D \Gamma \dot{\nu}=\Gamma^{T}\left\{\tau-C-H_{v}+\left(D[\Upsilon \dot{A}+\Gamma \dot{B}]-H_{m}\right) \Gamma \nu\right\}, \\
A D^{-1} A^{T} \epsilon=A D^{-1}\left\{\tau-C-H_{v}-H_{m} \Gamma \nu\right\}+\dot{A} \Gamma \nu
\end{gathered}
$$

The $\left(N_{12}-6\right)$ scalar equations comprising eq. (64) constitute the reduced order equations of motion for the closed chain system. The internal contact forces $\{\epsilon\}$ have been eliminated from eq. (64) which in turn are calculated as a function of the variables $(q, \nu, \tau)$ using eq. (65). Since $D$ is positive definite and $\Gamma$ and has full rank $\left(N_{12}-6\right)$, then $\left(\Gamma^{T} D \Gamma\right)$ is positive definite and therefore nonsingular. $\left(A D^{-1} A^{T}\right)$ is positive definite and nonsingular by a similar argument. Thus eqs. (64) and (65) can be solved for $\dot{\nu}$ and $\epsilon$, respectively. 
Given the separated form of the reduced order model, we can now proceed with the controller design. This is discussed next.

\section{CONTROL ARCHITECTURE}

The problem considered is to derive a control law for the $N_{12}$ joint torques $\tau=$ $\left[\tau_{1}^{T}, \tau_{2}^{T}\right]^{T}$ so that the variables $\{\epsilon, \nu\}$ quantifying the internal contact force- and positioncontrolled DOF can be controlled independently. This can be accomplished by applying the control law proposed in [31] to completely decouple eqs. (64) and (65). The composite control $\{\tau\}$ is the sum of an $\left(N_{12} \times 1\right)$ primary controller $\tau^{P}$ and an $\left(N_{12} \times 1\right)$ secondary controller $\tau^{s}$ which are defined by:

$$
\begin{gathered}
\tau^{p}=-\left(D[\Upsilon \dot{A}+\Gamma \dot{B}]-H_{m}\right) \Gamma \nu+C+H_{v}, \\
\tau^{s}=A^{T} \tau_{f}^{s}+D \Gamma \tau_{p}^{s} .
\end{gathered}
$$

In eq. $(67), \tau_{\rho}^{s}$ and $\tau_{p}^{s}$ are $(6 \times 1)$ and $\left(\left(N_{12}-6\right) \times 1\right)$ vectors, respectively, representing control variables to be determined.

The composite control $\left(\tau=\tau^{p}+\tau^{s}\right)$ defined by eqs. (66) and (67) is substituted into eqs. (64) and (65). The resulting equations, under the assumption of perfect knowledge of the nonlinear terms in the model, leads to the closed loop system:

$$
\begin{gathered}
\dot{\nu}=\tau_{p}^{s}, \\
\epsilon=\tau_{f}^{s}
\end{gathered}
$$

in which eq. (55) has been invoked. The derivation of eqs. (68) and (69) is based on the quantities $\left\{\left(\Gamma^{T} D \Gamma\right),\left(A D^{-1} A^{T}\right)\right\}$ being invertible. It was shown in Section 6 that these quantities are positive definite and therefore nonsingular.

Suppose $\tau_{p}^{s}$ is selected to servo the pseudovariable error, and $\tau_{f}^{s}$ for servoing the internal contact force error. Since eqs. (68) and (69) are completely decoupled, the secondary controller components $\tau_{p}^{s}$ and $\tau_{f}^{s}$ are non-interacting controllers for position and internal contact force, respectively.

It was claimed in [31] that the control architecture $\tau=\tau^{p}+\tau^{s}$ decoupled the control of the pseudovariables and an independent subset of the contact forces, namely those imparted by manipulator 2 . As shown here in section 3 , the modeling procedure in [31] unknowingly distributed the load such that $\epsilon=f_{c 2}$, i.e., the contact forces imparted by manipulator 2 are purely internal. The control law $\left(\tau=\tau^{p}+\tau^{s}\right)$ defined by eqs. (66) and $(67)$ in fact decouples the position- and internal force-controlled DOF. The physical insight into the decoupling was first identified in [33].

\section{CONCLUSION}

The paper has reviewed a method for modeling and controlling two serial link manipulators which mutually lift and transport a rigid body object in a three dimensional workspace. The system was viewed a single closed chain mechanism and it was assumed that there is no relative motion between the end effectors and object. A new vector variable $\epsilon$ which parameterizes the internal contact force controlled degrees of freedom was introduced. It was defined as a linear function of the contact forces that both manipulators impart to the object using eq. (9) . A family of solutions to the dynamic load distribution problem was obtained by solving the object's dynamical equations and eq. (9) for the contact forces. The motion inducing component of every member of the family was shown to be identical. The internal component of the general solution is a function of $\{\epsilon, M\}$, and each distinct member of the family is distinguished by the designer's choice for these variables. Three choices for matrix $M$ which transforms the contact forces to define $\epsilon$ in eq. (9) were suggested. 
The kinematic coupling effects between the manipulators due to the shared payload were modeled. First, the Cartesian velocity of the object at its center of mass was expressed as a linear function of the joint velocities of both manipulators. Then a set of six rigid body kinematic constraints restricting the values of the joint velocities was derived.

A rigid body dynamical model for closed chain system was first derived in the joint space. Our procedure for doing this requires expressing vector $Y$ in the object's dynamical equations in the joint space. We proposed here a generalization of our previous methods [30, 31] of accomplishing this where $Y=Y(\dot{q}, \ddot{q})$ is also an explicit function of the matrix $\Phi$. Our earlier results were shown to be specific choices for $\Phi$.

The last six equations in the joint space model are the kinematic acceleration constraints. By expressing the model in the pseudospace, it was shown that these last six equations become irrelevant. Linear transformations were applied to the upper $\left(N_{1}+N_{2}\right)$ equations in the model to obtain reduced order equations governing the motion of the system and a separate set of equations governing the internal components of the contact forces. Both sets are functions of the joint torques of both manipulators, but only the latter is a function of $\epsilon$. The control architecture originally proposed in [31] was applied to completely decouple the two sets of equations comprising the separated form of the model. As a result, the pseudovariables and the elements of $\epsilon$ are controlled independently.

\section{References}

[1] K. Laroussi, H. Hemami, and R.E. Goddard, "Coordination of Two Planar Robots in Lifting," IEEE Journal of Robotics and Automation, vol. 4, no. 1, pp. 77-85, February 1988.

[2] P. Chiacchio and S. Chiaverini, "User-Oriented Task Description for Cooperative Spatial Manipulators: Rolling Grasp" Robotics and Manufacturing, (Proc. Fifth Int'l Symposium on Robotics and Manufacturing (ISRAM'94), Maui, HI, August 14-18, 1994) edited by M. Jamshidi et. al., vol. 8, pp. 431-436, ASME Press, 1994.

[3] R. Bonitz and T. Hsia, "Robust Internal Force-tracking Impedance Control for Coordinated Multi-arm Manipulation - Theory and Experiments" Robotic and Manufacturing Systems, (Proc. World Automation Congress (WAC'96), May 28-30, 1996, Montpellier, France) edited by M. Jamshidi and F.G. Pin; TSI Press Series, 1996, pp. 111-118.

[4] R. Bonitz and T. Hsia, "The Effects of Computational Delays in Coordinated Multiplearm Manipulation Using Robust Internal Force-based Impedance Control" Robotic and Manufacturing Systems, (Proc. World Automation Congress (WAC'96), May 28-30, 1996, Montpellier, France) edited by M. Jamshidi and F.G. Pin; TSI Press Series, 1996, pp. 103-110.

[5] S. Schneider and R. Cannon, "Object Impedance Control for Cooperative Manipulation: Theory and Experimental Results" IEEE Trans. Robotics and Automation, vol. 8, no. 3, pp. 383-394, 1992.

[6] M. Uchiyama, T. Kitano, Y. Tanno, and K. Miyawaki, "Cooperative Multiple Robots to be Applied to Industries" Robotic and Manufacturing Systems, (Proc. World Automation Congress (WAC'96), May 28-30, 1996, Montpellier, France) edited by M. Jamshidi and F.G. Pin; TSI Press Series, 1996, vol. 3, pp. 759-764.

[7] M. Uchiyama, X. Delebarre, H. Amada, and T. Kitano, "Optimum Internal Force Control for Two Cooperative Robots to Carry an Object", Intelligent Automation and Soft Computing, (Proc. World Automation Congress (WAC'94), Maui, HI, August 1417, 1994) vol. 2, pp. 111-116, TSI Press Series, 1994. 
[8] M. Uchiyama and P. Dauchez, "Symmetric kinematic formulation and non-master slave coordinated control of two-arm robots", Advanced Robotics, vol. 7, no. 4, pp. 361-383, 1993.

[9] P. Chiacchio and S. Chiaverini, "PD-Type Control Schemes For Cooperative Manipulator Systems," Intelligent Automation and Soft Computing, (journal) vol. 2, no. 1, pp. 65-72, 1996.

[10] O. Khatib, K. Yokai, K. Chang, D. Ruspini, R. Holmberg, and A. Casal, "Cooperative Tasks in Multiple Mobile Manipulation Systems," Robotics and Manufacturing, (Proc. Sixth Int'l Symposium on Robotics and Manufacturing (ISRAM'96), May 28-30, 1996, Montpellier, France) edited by M. Jamshidi et. al., vol. 6, pp. 345-350, ASME Press, 1996.

[11] D. Williams and O. Khatib, "Modeling and Control of Internal Force Dynamics in MultiGrasp Manipulation", Robotics and Manufacturing, (Proc. Fifth Int'l Symposium on Robotics and Manufacturing (ISRAM'94), Maui, HI, August 14-18, 1994) edited by M. Jamshidi et. al., vol. 5, pp. 735-741, ASME Press, 1994.

[12] P. Hsu, "Adaptive Internal Force Control of a Two Manipulator System," Robotics and Manufacturing, (Proc. Fifth Int'l Symposium on Robotics and Manufacturing (ISRAM'94), Maui, HI, August 14-18, 1994) edited by M. Jamshidi et. al., vol. 5, pp. 151156, ASME Press, 1994.

[13] P. Hsu, "Coordinated Control of Multiple Manipulator Systems", IEEE Trans. Robotics and Automation, vol. 9, no. 4, pp. 400-410, August, 1993.

[14] D.J. Cox and D. Tesar, "Development System Environment For Dual-Arm Robotic Operations," Robotics and Manufacturing, (Proc. Fifth Int'l Symposium on Robotics and Manufacturing (ISRAM'94), Maui, HI, August 14-18, 1994) edited by M. Jamshidi et. al., vol. 5, pp. 61-66, ASME Press, 1994.

[15] D.J. Cox and D. Tesar, "Cooperative Dual-Arm Robotic Operations with Fixture Interaction," Intelligent Automation and Soft Computing, (Proc. World Automation Congress (WAC'94), Maui, HI, August 14-17, 1994) vol. 2, pp. 439-444, TSI Press Series, 1994.

[16] F. Caccavale and J. Szewczyk, "Experimental Results of Operational Space Control on a Dual-Arm Robot System," Robotics and Manufacturing, (Proc. Sixth Int'l Symposium on Robotics and Manufacturing (ISRAM'96), May 28-30, 1996, Montpellier, France) edited by M. Jamshidi et. al., vol. 6, pp. 121-126, ASME Press, 1996.

[17] J.T. Wen and K. Kreutz Delgado, "Motion and Force Control of Robotic Manipulators," Automatica, vol. 28, pp. 729-743, 1992.

[18] K. Kreutz and A. Lokshin, "Load Balancing and Closed Chain Multiple Arm Control," American Control Conf., June 1988, Atlanta, GA, vol. 3, pp. 2148-2155.

[19] I.D. Walker, R.A. Freeman, and S.I. Marcus, "Analysis of Motion and Internal Loading of Objects Grasped by Multiple Cooperating Manipulators," Int'l J. of Robotics Research, August 1991, vol. 10, no. 4, pp. 396-409.

[20] I.D. Walker, S.I. Marcus, and R.A. Freeman, "Distribution of Dynamic Loads for Multiple Cooperating Robot Manipulators," J. Robotic Systems, vol. 6, no. 1, pp. 35-47, January 1989.

[21] F. Pfeifer, "Cooperating Fingers - A Special Form of Cooperating Robots," Robotic and Manufacturing Sysiems, (Proc. World Automation Congress (WAC'96), May 2830, 1996, Montpellier, France) edited by M. Jamshidi and F.G. Pin; TSI Press Series, 1996, pp. $639-643$. 
[22] W. Nguyen and J.K. Mills "Fixtureless Assembly: Multi-Robot Manipulation of Flexible Payloads," Robotics and Manufacturing, (Proc. Sixth Int'l Symposium on Robotics and Manufacturing (ISRAM'96), May 28-30, 1996, Montpellier, France) edited by M. Jamshidi et. al., vol. 6, pp. 661-666, ASME Press, 1996.

[23] M.E. Pittelkau, "Adaptive Load Sharing Force Control for Two-Arm Manipulators," IEEE Int'l Conf. Robotics and Automation, vol. 1, pp. 498-503, Philadelphia, PA, April 24-29, 1988.

[24] Y.-R. Hu and A.A. Goldenberg, "An Adaptive Approach to Motion and Force Control of Multiple Coordinated Robot Arms," IEEE Int'l Conf. Robotics and Automation, vol. 2, pp. 1091-1096, Scottsdale, AZ, May 14-19, 1989.

[25] Y. Nakamura, K. Nagai, and T. Yoshikawa, "Dynamics and Stability in Coordination of Multiple Robotic Mechanisms," Int'l J. of Robotics Research, April 1989, Vol. 8, No. 2, pp. 44-61.

[26] Y.D. Shin and M.J. Chung, "An Optimal Force Distribution Scheme for Cooperating Multiple Robot Manipulators", Robotica, pp. 49-60, Vol. 11, Part 1, Jan.-Feb. 1993.

[27] M.H. Choi, B.H. Lee, and M.S. Ko, "Optimal Load Distribution for Two Cooperating Manipulators Using a Force Ellipsoid", Robotica, pp. 61-72, Vol. 11, Part 1, Jan.-Feb. 1993.

[28] L.T. Wang and M.J. Kuo, "Dynamic Load Carrying Capacity and Inverse Dynamics of Multiple Cooperating Manipulators", IEEE Trans. Robotics and Automation, pp. 7177, Vol. 10, no. 1, February 1994.

[29] M.A. Unseren, "Determination of Contact Forces for Two Manipulators Mutually Lifting a Rigid Object Using a Technique of Dynamic Load Distribution, "Intelligent Automation and Soft Computing, (journal) Vol. 2, No. 1, 1996, pp. 49-63.

[30] M.A. Unseren, "A New Technique for Dynamic Load Distribution when Two Manipulators Mutually Lift a Rigid Object. Part 1: The Proposed Technique (pp. 359-365); Part 2: Derivation of Entire System Model and Control Architecture (pp. 367-372)"; Intelligent Automation and Soft Computing, Trends in Research, Development, and Applications, (Proc. World Automation Congress (WAC '94), August 14-17, 1994, Maui, HI) edited by M. Jamshidi, etc.; TSI Press Series, 1994.

[31] M.A. Unseren, "Rigid body dynamics and decoupled control architecture for two strongly interacting manipulators," Robotica, 1991, vol. 9, part 4, pp. 421-430.

[32] M.A. Unseren, "A Review of a Method for Dynamic Load Distribution, Dynamical Modeling, and Explicit Internal Force Control When Two Serial Link Manipulators Mutually Lift and Transport a Rigid Body Object," Oak Ridge National Laboratory Technical Report \# ORNL/TM-13429, 1997 (in press), Oak Ridge, TN, USA.

[33] M.A. Unseren, "A Rigid Body Model and Decoupled Control Architecture For Two Manipulators Holding a Complex Object," Robotics and Autonomous Systems, Vol. 10, No. 2-3, 1992, pp. 115-131.

[34] J.Y.S. Luh and Y.F. Zheng, "Constrained Relations Between Two Coordinated Industrial Robots For Motion Control," International Journal of Robotics Research, Fall 1987, vol. 6, no. 3, pp 60-70.

[35] M.A. Unseren, "An Approach to Modeling a Kinematically Redundant Closed Chain System Using Pseudovelocities," Robotics and Manufacturing, (Proc. Sixth Int'l Symposium on Robotics and Manufacturing (ISRAM'96), May 28-30, 1996, Montpellier, France) edited by M. Jamshidi et. al., vol. 6, pp. 843-850, ASME Press, 1996.

[36] B. Noble and J.W. Daniel, Applied Linear Algebra, 2nd ed., Prentice-Hall, Inc., 1977. 
[37] H. Goldstein, Classical Mechanics, 2nd edition, Addison-Wesley, 1980.

[38] H. Hemami and F.C. Weimer, "Modeling of Nonholonomic Dynamic Systems with Applications," ASME J. of Applied Mechanics, March 1981, vol. 48, pp. 177-182.

[39] R.K. Kankaanranta and H.N. Koivo, "Dynamics and Simulation of Compliant Motion of a Manipulator," IEEE J. Robotics and Automation, April 1988, vol. 4, no. 2, pp. 163173.

[40] D.T. Finkbeiner, Introduction to Matrices and Linear Transformations, 3rd ed., W.H. Freeman and Company, 1978.

[41] F.R. Gantmacher, Lectures in Analytical Mechanics, USSR: Mir Publishers, chap. 1, sec. $10,1975$.

[42] H. Hemami, "A Feedback On-Off Model of Biped Dynamics," IEEE Trans. Systems, Man, and Cybernetics, July 1980, vol. SMC-10, no. 7, pp. 376-383, sec. IV.

[43] J.W. Kamman and R.L. Huston, "Dynamics of Constrained Multibody Systems," ASME Journal of Applied Mechanics, December 1984, Vol. 51, pp. 899-903. 